\title{
IN VITRO ANALYSIS OF UNIVERSALLY UTILIZED IMPLANT RESTORATIVE DENTAL MATERIAL'S IMPACT ON THE ARCHITECTURAL STABILITY OF GINGIVAL FIBROBLASTS IN THE PRESENCE OF A COMMON ENDOTOXIN
}

\author{
Angelia D. Garner ${ }^{1}$, Michelle A. Tucci ${ }^{2}$, Hamed A. Benghuzzi ${ }^{3}$ \\ ${ }^{1}$ University of Mississippi Medical Center, School of Dentistry, Jackson, MS 39216 \\ ${ }^{2}$ University of Mississippi Medical Center, School of Medicine , Jackson, MS 39216 \\ Global Training Institute, Flowood, MS
}

Corresponding Author: Angelia D. Garner

Address: 2500 North State St., Jackson, MS 39216

Tel: 1-601-984-6347

Email: adgarner@umc.edu

https://doi.org/10.34107/BiomedSciInstrum.57.0486

\begin{abstract}
Dental implants have been utilized in the last several decades to replace missing teeth. Various factors may result in the loss of teeth. The most common causes of tooth loss are often caries or periodontal disease. The use of a dental implant restored with a porcelain fused to metal crown is often the standard. The purpose of this study was to assess the architectural integrity of gingival fibroblasts at the cellular level when exposed to universally utilized restorative dental material; porcelain, in the presence of a periodontal pathogen, Porphyromonas gingivalis lipopolysaccharide (LPS-PG). Human gingival fibroblasts were exposed to Porcelain $(.1 \mathrm{~g})$ in combination with $L P S-P G(10 \mu \mathrm{L})$, at 24, 48, and 72 hour durations. When assessing for cellular metabolic activity and viability, no significant differences were noted between the control and experimental groups. Contrastingly, when assessing for oxidative stress, the experimental groups were statistically significantly different from the control at the 48 and 72 hour phases $(\mathrm{P}<0.001)$. H\&E staining of the experimental groups showed irregular shaped cells with loss of density, vacuolization, coarse cytoplasm, and hyperchromatic nuclei.
\end{abstract}

Keywords: Dental restorations, Gingival fibroblasts, Porphyromas gingivalis, Porcelain

\section{INTRODUCTION}

Medical devices such as implants have been utilized in various medical disciplines to restore the loss of tissues and their functions [1]. In the field of dentistry, implants have become a vital component of prosthetic rehabilitation for missing teeth. Implants are used to replace teeth loss due to many factors. The leading causes of tooth loss are dental caries and periodontal disease worldwide.

Caries and periodontal disease are chronic infectious diseases found in the oral cavity. When left untreated or poorly managed; both, dental caries and periodontal disease can lead to infection, pain, and tooth loss. It is estimated that $90 \%$ of adults will have a carious lesion in their permanent dentition [2]. With regards to periodontal disease, it has been estimated that more than one-third of the adult population suffers from chronic-moderate to severe periodontitis [3]. Unlike with caries, the loss of the tooth does 